\title{
Ergodic Properties of the Equilibrium Process Associated with Infinitely Many Markovian Particles
}

By

Tokuzo ShIGA* and Yoichiro TAKAHASHI**

\section{§Q. Hntrodnetion}

Consider a system of independent identically distributed Markov processes which have an invariant measure $\lambda$. It is known that if each process starts from each point of a $\lambda$-Poisson point process at time zero, these particles are $\lambda$-Poisson distributed at every later time $t>0$ [1].

In the present paper we are concerned with the ergodic properties of the stationary processes obtained from such a system of particles, which is called the equilibrium process. Sinai's ideal gas model is a special example of the equilibrium processes [4]. In $\S 1$ we will give some preliminaries and the definition of the equilibrium process, and $\S 2$ is devoted to the study of the ergodic properties (metrical transitivity, mixing properties and pure nondeterminism) of the equilibrium processes. In $\S 3$ we will discuss the Bernoulli property in the strong sense of the shift flow $\left\{\Theta_{t}\right\}_{-\infty<t<\infty}$ defined in $\$ 1$. The shift flow induced by the eqilibrium process is a factor flow of $\left\{\Theta_{t}\right\}$. In $\$ 4$ we prove a central limit theorem. Finally the authors would like to express their hearty gratitude to Professor H. Tanaka for his valuable advice.

\section{§1。 Proeliminimaries}

Let $\left(X, \mathscr{B}_{X}, \lambda\right)$ be a $\sigma$-finite measure space, and denote by $\mathscr{K}(X)$ a

Communicated by H. Araki, April 24, 1973.

* Department of Mathematics, Nara Women's University, Nara.

** College of General Education, University of Tokyo, Tokyo. 
family of all the counting measures on $X$, i.e. each element of $\mathscr{K}(X)$ is an integer-valued measure with a countable set as its support. $\mathscr{K}(X)$ is equipped with a $\sigma$-field $\mathscr{G}$ which is generated by $\{\rho \in \mathscr{K}(X): \rho(A)=n\}$, $n \geqq 0, A \in \mathscr{B}_{X}$. An element $\rho$ of $\mathscr{K}(X)$ is represent by $\rho=\sum_{i} \delta_{x i}$ where $\delta_{x}(A)=1$ if $x \in A$ and $\delta_{x}(A)=0$ if $x \notin A$.

Definition $\mathbb{1}_{\text {.1. }}$ Let $\Pi_{\lambda}$ be a probability measure on $(\mathscr{K}(X), \mathscr{G}) . \quad \Pi_{\lambda}$ is $\lambda$-Poisson point process if it satisfies the following conditions;

(1.1) for any disjoint system $A_{1}, \ldots, A_{n}$ of $\mathscr{B}_{X}$ such that $\lambda\left(A_{i}\right)<+\infty_{i=1, \ldots, n}$ $\rho\left(A_{1}\right), \ldots, \rho\left(A_{n}\right)$ are independent random variables on $\left(\mathscr{K}(X), \mathscr{G}, \mathbb{I}_{\lambda}\right)$ and $\Pi_{\lambda}\left\{\rho ; \rho\left(A_{i}\right)=n\right\}=\frac{\left[\lambda\left(A_{i}\right)\right]^{n}}{n !} \exp \left[-\lambda\left(A_{i}\right)\right], i=1, \ldots, n$.

Here we summarize some elementary facts on $\lambda$-Poisson point process.

\section{Lemma 1.2.}

(a) For any $\sigma$-finite measure space $\left(X, \mathscr{B}_{X}, \lambda\right)$ there exists a $\lambda$-Poisson point process.

(b) A probability measure $\Pi_{\lambda}$ on $(\mathscr{K}(X), \mathscr{G})$ is a $\lambda$-Poisson point process if and only if

(1.2) $\int e^{-<\varphi, \rho>} \Pi_{\lambda}(d \rho)=e^{-<1-e^{-\varphi}, \lambda>}$ 1) for every non-negative measurable function $\varphi$ on $\left(X, \mathscr{B}_{X}\right)$,

and moreover (1.2) is equivalent to the following condition;

(1.3) $\int e^{i<\varphi, \rho>} \Pi_{\lambda}(d \rho)=e^{-<1-e^{i \varphi}, \lambda>}$ for every $\lambda$-integrable function $\varphi$.

For each $A$ of $\mathscr{B}_{X}$, denote by $\mathscr{G}(A)$ the $\sigma$-field generated by $\{\rho \in \mathscr{K}(X)$; $\rho(B)=n\}, n \geqq 0, B \in \mathscr{B}_{X}, B \subset A$.

\section{Lemma 1.3.}

(a) If $A_{1}, \ldots, A_{n}$ are mutually disjoint, $\mathscr{G}\left(A_{1}\right), \ldots, \mathscr{G}\left(A_{n}\right)$ are mutually independent $\sigma$-fields w.r.t. $\Pi_{\lambda}$.

1) For a function $\varphi$ and a measure $\lambda<\varphi, \lambda\rangle=\int \varphi(x) \lambda(d x)$. 
(b) If $\left\{A_{n}\right\} \subset \mathscr{B}_{X}$ is non-increasing and $\bigcap_{n} A_{n}=\phi,\left\{\mathscr{G}\left(A_{n}\right)\right\}$ is also nonincreasing and $\bigcap_{n} \mathscr{G}\left(A_{n}\right)=\{\phi, \mathscr{K}(X)\}\left(\bmod . \Pi_{\lambda}\right)$.

Next, we define the equilibrium processes associated with Markovian particles.

Let $X$ be a locally compact separable Hausdorff space and $\mathscr{B}_{X}$ be the topological Borel field of $X$. Denote by $\mathscr{W}$ the path space of $X$, that is, each element of $\mathscr{W}$ is a $X$-valued right continuous function with left limit defined on $(-\infty, \infty)$, and define the shift operators $\left\{\theta_{t}\right\}_{-\infty<t<\infty}$ of $\mathscr{W}$ as usual; $\left(\theta_{t} f\right)_{s}=f_{t+s}$ for each $f$ of $\mathscr{W}$.

Put $S=\mathscr{K}(X)$ and $\Omega=\mathscr{K}(\mathscr{W})$. Denote by $\left\{\Theta_{t}\right\}_{-\infty<t<\infty}$ the shift operators on $\Omega$ induced by the shift operators $\left\{\theta_{t}\right\}_{-\infty<t<\infty}$ on $\mathscr{W}$, i.e.

$$
\Theta_{t} \omega=\sum_{i} \delta_{\theta_{t} f^{i}} \quad \text { if } \quad \omega=\sum \delta_{f^{i}}
$$

Define $S$-valued process $\left\{\xi_{t}(\omega)\right\}_{-\infty<t<\infty}$ on $\Omega$ as follows;

$$
\xi_{t}(\omega)=\sum_{i} \delta_{f_{t}^{i}} \quad \text { if } \quad \omega=\sum_{i} \delta_{f}
$$

Then $\xi_{t}(\omega)$ is right continuous in $t$ in a natural topology.

In our situation a motion of one particle is given as a Markov process on $X$ and denote by $\left\{P_{t}(x, d y)\right\}$ its transition probabilities.

\section{Assumption.}

$\left\{P_{t}(x, d y)\right\}$ is a conservative Feller Markov process and have a Radon invariant measure $\lambda$, that is, $\left\{P_{t}(x, d y)\right\}$ induces a semi-group of contraction operators $\left\{T_{t}\right\}$ on $C_{\infty}(X)$, and $\int T_{t} f(x) \lambda(d x)=\int f(x) \lambda(d x)$ for every $f$ of $C_{0}(X){ }^{2}{ }^{2}$

Under this assumption $\left\{T_{t}\right\}$ is, also, a semi-group of contraction operators on $L^{2}\left(X, \mathscr{B}_{X}, \lambda\right)$.

Lemma $\mathbb{1} . \mathbb{A}$. There is only one $\sigma$-finite measure $Q$ on $(\mathscr{W}, \mathscr{B} \mathscr{\mathscr { V }})^{3)}$ such that

2) $C_{\infty}(X)$ is the family of all the continuous functions vanishing at infinity, and $C_{0}(X)$ is the family of all the continuous functions with compact supports.

3) $\mathscr{B} \mathscr{\mathscr { V }}$ is the $\sigma$-algebra generated by all the cylindrical subsets of $\mathscr{W}$. 


$$
\begin{aligned}
& \text { for }-\infty<t_{1}<t_{2}<\cdots<t_{n}<+\infty \text { and }\left\{A_{i}\right\}_{i=1,2 \ldots, n} \\
& Q\left[f ; f_{t_{1}} \in A_{1}, f_{t_{2}} \in A_{2}, \ldots, f_{t_{n}} \in A_{n}\right] \\
& =\int_{A_{1}} \lambda\left(d x_{1}\right) \int_{A_{2}} P_{t_{2}-t_{1}}\left(x_{1}, d x_{2}\right) \cdots \int_{A_{n}} P_{t_{n}-t_{n-1}},\left(x_{n-1}, d x_{n}\right) .
\end{aligned}
$$

In particular $Q$ is $\left\{\theta_{t}\right\}$-invariant.

Denote by $\mathbb{B}$ the $\sigma$-field generated by $\{\omega \in \Omega ; \omega(A)=n\}, n \geqq 0, A \in \mathscr{B}_{X}$ and put $\mathbb{P}=\Pi_{Q}(Q$-Poisson point process). We consider $(\Omega, \mathbb{R}, \mathbb{P})$ as our basic probability space.

Proposition $1.5 . \quad\left\{\Omega, \mathbb{B},\left\{\xi_{t}\right\}_{-\infty<t<\infty}\right\}$ is a right-continuous Markov stationary process with $\Pi_{\lambda}$ as its absolute law.

Proof. It is sufficient to prove the following formula;

(1.7) for $-\infty<t_{1}<t_{2}<\cdots<t_{n}<\infty$ and $\left\{\varphi_{i}\right\} \geqq 0$ measurable functions on $X$

$\mathbb{E}\left[e^{-\left\langle\varphi_{1}, \xi_{t_{1}}\right\rangle} \ldots e^{-\left\langle\varphi_{n}, \xi_{t}\right\rangle}\right]=\mathbb{E}\left[e^{-\left\langle\varphi_{1}, \xi_{t_{1}}\right\rangle} \ldots e^{-\left\langle\varphi_{n-1}, \xi_{t_{n}-1}\right\rangle} e^{<\log T_{t_{n}-t_{n-1}} e^{\left.-\varphi_{n}, \xi_{t_{n-1}}\right\rangle}}\right]_{\cdot}{ }^{4)}$

Put $\bar{\Phi}(f)=\sum_{i=1}^{n} \varphi_{i}\left(f_{t_{i}}\right)$.

The left-hand side of $(1.7)=\mathbb{E}\left[e^{-\langle\tilde{\omega}, \omega\rangle}\right]=\exp -\left\langle 1-e^{-\bar{\omega}}, Q\right\rangle$

$$
\begin{aligned}
& =\exp -\int \lambda\left(d x_{1}\right) \int P_{t_{2}-t_{1}}\left(x_{1}, d x_{2}\right) \cdots \int P_{t_{n}-t_{n-1}}\left(x_{n-1}, d x_{n}\right)\left[1-e^{-\sum_{1}^{n} \varphi_{i}\left(x_{i}\right)}\right] \\
& =\exp -\int \lambda\left(d x_{1}\right) \int P_{t_{2}-t_{1}}\left(x_{1}, d x_{2}\right) \cdots \\
& \quad \cdots \int P_{t_{n-1}-t_{n-2}}\left(x_{n-2}, d x_{n-1}\right)\left[1-e^{-\sum_{1}^{n-1} \varphi_{i}\left(x_{i}\right)} T_{t_{n-t_{n-1}}} e^{-\varphi_{n}}\left(x_{n-1}\right)\right] \\
& =\mathbb{E}\left[e^{\left.-<\varphi_{1}, \xi_{t_{1}}>\ldots e^{-<\varphi_{n-1}-\log T_{t_{n-t}-1}} e^{-\varphi_{n}, \xi_{t_{n-1}}>}\right]}\right. \\
& =\text { the right-hand side of }(1.7) .
\end{aligned}
$$

In particular $\left.\mathbb{E}\left[e^{-\left\langle\varphi, \xi_{t}\right\rangle}\right]=\exp -<1-e^{-\varphi}, \lambda\right\rangle$.

Defimition 1.6. The Markov stationary process ( $\mathcal{Q}, \mathbb{B}, \mathbb{P} ;\left\{\xi_{t}\right\}_{-\infty<t<\infty}$ )

4) $\boldsymbol{E}$ denotes the expectation by $\boldsymbol{P}$. 
is called the equilibrium process associated with $\left[\left\{T_{t}\right\}, \lambda\right]$.

The following calculations are immediate from (1.7).

\section{Proposition 1.7.}

(i) $\mathbb{E}\left[e^{-\left\langle\varphi, \xi_{t}\right\rangle} \mid \xi_{s}\right]=e^{\left.<\log T_{t-s} e^{-\varphi}, \xi_{s}\right\rangle} \quad$ for ${ }^{\forall} \varphi \geqq 0$ and $s<t$.

(ii) $\left.\left.\mathbb{E}\left[<\varphi, \xi_{t}\right\rangle \mid \xi_{s}\right]=<T_{t-s} \varphi, \xi_{s}\right\rangle$ for ${ }^{\forall} \varphi \in L^{2}\left(X, \mathscr{B}_{X}, \lambda\right)$.

(iii) $\mathbb{E}\left[<\varphi, \xi_{t}><\psi, \xi_{t}>\mid \xi_{s}\right]=<T_{t-s} \varphi, \xi_{s}><T_{t-s} \psi, \xi_{s}>$

$$
+<T_{t-s}(\varphi \psi), \xi_{s}>-<T_{t-s} \varphi \cdot T_{t-s} \psi, \xi_{s}>
$$

for ${ }^{\forall} \varphi, \phi \in L^{2}\left(X, \mathscr{B}_{X}, \lambda\right) \cap L^{1}\left(X, \mathscr{B}_{X}, \lambda\right)$.

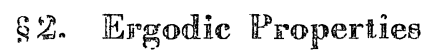

In this section we discuss the ergodic properties of the equilibrium processes.

Proposition 2.1. The following (i) (iii) are equivalent.

(i) $\left(\Omega, \mathbb{P},\left\{\xi_{t}\right\}_{-\infty<t<\infty}\right)$ is metrically transitive.

(ii) $\lim _{t \rightarrow \infty} \frac{1}{t} \int_{0}^{t} \int_{K} P_{s}(x, K) \lambda(d x) d s=0 \quad$ for every compact subset $K$ of $X$.

(iii) $\lim _{t \rightarrow \infty} \frac{1}{t} \int_{0}^{t}\left(T_{s} f, g\right)_{L^{2}(\lambda)} d s=0 \quad$ for all $f$ and $g$ of $L^{2}(X, \lambda)$

Proof. It is easy to show the equivalence of (ii) and (iii). Moreover (i) is equivalent to

$$
\begin{aligned}
& \text { (2.1) } \lim _{t \rightarrow \infty} \frac{1}{t} \int_{0}^{t} \mathbb{E}\left[c^{-\sum_{i}^{n}<\varphi_{1}, \xi_{s}>}>e^{-\sum_{1}^{m}-\phi_{1}, \xi_{2},+\cdots}\right] d u \\
& =\mathbb{E}\left[e^{-\sum_{1}^{n}<\varphi_{i}, \xi_{s_{i}}>}\right] \mathbb{E}\left[e^{-\sum_{1}^{m}\left\langle\psi j \cdot \xi_{r_{j}}\right\rangle}\right], \\
& \text { for any }-\infty<s_{1}<s_{2}<\cdots<s_{n}<\infty,-\infty<r_{1}<r_{2}<\cdots<r_{m} \text { and any } \\
& \left.\left\{\varphi_{i}\right\}_{i=1, \ldots, n},\left\{\phi_{j}\right\}_{j=1, \ldots, m} \text { of } C_{0}^{+}(X) .{ }^{5}\right)
\end{aligned}
$$

However it suffices to prove (2.1) for $m=1$ because of the Markov property of $\left\{\xi_{t}\right\}$. For $s_{n}<r+u$,

5) $C_{0}^{+}(X)$ is the family of non-negative elements of $C_{0}(X)$. 


$$
\begin{aligned}
& \mathbb{E}\left[e^{-\sum_{1}^{n}\left\langle\xi_{i}, \xi_{s_{i}}\right\rangle} e^{-\left\langle\psi, \xi_{r+u}\right\rangle}\right] \\
& =\exp -\int \lambda(d x)\left(1-e^{-\varphi_{1}} T_{s_{2}-s_{1}} e^{-\varphi_{2}} \cdots e^{-\varphi_{n}} T_{r+u-s_{n}} e^{-\psi}\right) \\
& =\exp -\int \lambda(d x)\left(\left(1-e^{-\varphi_{1}} T_{s_{2}-s_{1}} e^{-\varphi_{2} \ldots T_{s_{n}-s_{n-1}} e^{-\varphi_{n}}}\right)\right. \\
& \left.+e^{-\varphi_{1}} T_{s_{2}-s_{1}} e^{-\varphi_{2}} \ldots T_{s_{n}-s_{n-1}} e^{-\varphi_{n}} T_{r+u-s_{n}}\left(1-e^{-\phi}\right)\right) \\
& =\mathbb{E}\left[e^{-\sum_{1}^{n}\left\langle\varphi_{\imath}, \xi_{s_{2}}\right\rangle}\right] \mathbb{E}\left[e^{-\left\langle\psi_{1}, \xi_{r}\right\rangle}\right] \times \\
& \exp \int \lambda(d x)\left[I-e^{-\varphi_{1}} T_{s_{2-s_{1}}} \cdots T_{s_{n}-s_{n-1}} e^{-\varphi_{n}}\right] T_{r+u-s_{n}}\left(1-e^{-\psi}\right)
\end{aligned}
$$

Therefore,

(2.2) $\lim _{t \rightarrow \infty} \frac{1}{t} \int_{0}^{t} \mathbb{E}\left[e^{-\sum_{i=1}^{n}\left\langle\varphi_{i}, \xi_{s_{2}}\right\rangle} e^{-\left\langle\psi, \xi_{r+u}\right\rangle}\right] d u=\mathbb{E}\left[e^{-\sum_{1}^{n}\left\langle\varphi_{i}, \xi_{s_{i}}\right\rangle}\right] \mathbb{E}\left[e^{-\left\langle\psi, \xi_{r}\right\rangle}\right]$ is equivalent to

(2.3) $\lim _{t \rightarrow \infty} \frac{1}{t} \int_{0}^{t} \exp \left[\int \lambda(d x)\left[I-e^{-\varphi_{1}} T_{s_{2}-s_{1}} e^{-\varphi_{2}} \ldots T_{s_{n}-s_{n-1}} e^{-\varphi_{n}}\right] \times\right.$

$$
\left.T_{u}\left(1-e^{-\psi}\right)\right] d u=1
$$

or

(2.4) $\lim _{t \rightarrow \infty} \frac{1}{t} \int_{0}^{t}\left[\int \lambda(d x)\left[I-e^{-\varphi_{1}} T_{s_{2}-s_{1}} e^{-\varphi_{2}} \ldots T_{s_{n}-s_{n-1}} e^{-\varphi_{n}}\right]\right.$

$$
\left.T_{u}\left(1-e^{-\phi}\right)\right] d u=0
$$

Note $1-e^{-\varphi_{1}} T_{s_{2}-s_{1}} e^{-\varphi_{2}} \ldots T_{s_{n}-s_{n-1}} e^{-\varphi_{n}} \in L^{2}(X, \lambda)$ and $\left(1-e^{-\psi}\right) \in L^{2}(X, \lambda)$.

Hence (iii) implies (i). On the other hand it is obvious (2.4) implies (ii) by putting $n=1, \varphi_{1}=\psi \in C_{0}^{+}(X)$.

Corollary 2.2. If $\left\{\xi_{t}\right\}_{-\infty<t<\infty}$ is metrically transitive, then $\lambda(X)=\infty$.

Proposition 2.3. The following three statements are equivalent.

(i) $\left(\Omega, \mathbb{B}, \mathbb{P} ;\left\{\xi_{t}\right\}_{-\infty<t<\infty}\right)$ has the mixing property. 
(ii) $\lim _{t \rightarrow \infty} \int_{K} \lambda(d x) P_{t}(x, K)=0 \quad$ for every compact subset $K$ of $X$.

(iii) $\lim _{t \rightarrow \infty}\left(T_{t} f, g\right)_{L^{2}(X, \lambda)}=0 \quad$ for all $f$ and $g$ of $L^{2}(X, \lambda)$.

Since the proof of Proposition 2.3 can be carried out by the similar method as Proposition 2.1, it is omitted.

Next, we consider the pure non-determinism of the equilibrium processes. In general, let $\left(\Omega, \mathscr{F}, P ;\left\{z_{t}\right\}_{-\infty<t<\infty}\right)$ be a Markov stationary process on $X$ associated with $\left\{P_{t}(x, d y), \mu(d x)\right\}$, where $P_{t}(x, d y)$ is a transition probability measure and $\mu$ is an invariant probability measure. Then the following criterion for the pure non-determinism is applicable.

Lemma 2.4. ( $\left.\Omega, \mathscr{F}, P ;\left\{z_{t}\right\}_{-\infty<t<\infty}\right)$ is purely non-deterministic i.e. $\cap \mathscr{F}_{t}(z)=\{\phi, \Omega\}\left(\bmod . P\right.$ ) where $\mathscr{F}_{t}(z)$ is the $\sigma$-field generated by $\left\{z_{s} ; s \leqq t\right\}$, if and only if

$$
\lim _{t \rightarrow \infty} \int\left[\int P_{t}(x, d y) f(y)-\int \mu(d y) f(y)\right]^{2} \mu(d x)=0 \quad \text { for } \quad{ }^{\forall} f \in L^{2}(X, \mu) .
$$

This lemma can be found in $[6]$.

Proposition 2.5. The following three statements are equivalent.

(i) $\left(\Omega, \boldsymbol{B}, \boldsymbol{P} ;\left\{\xi_{t}\right\}_{-\infty<t<\infty}\right)$ is purely non-deterministic.

(ii) $\lim _{t \rightarrow \infty} \int_{X} \lambda(d x)\left[P_{t}(x, K)\right]^{2}=0 \quad$ for every compact subset $K$ of $X$.

(iii) $\lim _{t \rightarrow \infty}\left\|T_{t} f\right\|_{L^{2}(X, \lambda)}=0 \quad$ for every $f$ of $L^{2}(X, \lambda)$.

Proof. By Lemma 2.4 (i) is equivalent to

$$
\lim _{t \rightarrow \infty} \mathbb{E}\left[\left(\mathbb{E}\left[e^{-\left\langle\varphi, \xi_{t}\right\rangle} \mid \xi_{0}\right]-\mathbb{E}\left[e^{-\left\langle\xi_{0}, \varphi\right\rangle}\right]\right)^{2}\right]=0 \quad \text { for }{ }^{\forall} \varphi \in C_{0}^{+}(X)
$$

Using Proposition 1.7 and Poisson properties, we have

$$
\begin{aligned}
& \mathbb{E}\left[\left(\mathbb{E}\left[e^{-\left\langle\varphi, \xi_{t}\right\rangle} \mid \xi_{0}\right]-\mathbb{E}_{[}\left[e^{-\left\langle\xi_{0}, \varphi>\right.}\right]\right)^{2}\right]=\mathbb{E}\left[\left(e^{\left.<\log T_{t} e^{-\varphi}, \xi_{0}\right\rangle}-e^{-<1-e^{-\varphi}, \lambda>}\right)^{2}\right] \\
& =\mathbb{E}\left[e^{\left.<\log \left(T_{t} e^{-\varphi}\right)^{2}, \xi_{0}\right\rangle}-e^{\left.-2<1-e^{-\varphi}, \lambda\right\rangle}\right]=e^{-\left\langle 1-\left(T_{t} e^{-\varphi}\right)^{2}, \lambda\right\rangle}-e^{\left.-2<1-e^{-\varphi}, \lambda\right\rangle} \\
& =\left(e^{\left.<\left(T_{t}\left(1-e^{-\varphi}\right)\right)^{2}, \lambda\right\rangle}-1\right) e^{\left.-2<1-e^{-\varphi}, \lambda\right\rangle} .
\end{aligned}
$$

In the last equality we used $\left\langle T_{t}\left(1-e^{-\varphi}\right), \lambda\right\rangle=\left\langle 1-e^{-\varphi}, \lambda\right\rangle$. Therefore 
the equivalence of (2.6) and the statement (ii) is obvious. Moreover the equivalence of (ii) and (iii) is trivial.

Proposition 2.6. ( $\left.\Omega, \mathbb{B}, \mathbb{P} ;\left\{\xi_{t}\right\}_{-\infty<t<\infty}\right)$ is purely non-deterministic if and only if

$\mathbb{E}\left[\xi_{t} \mid \xi_{0}\right]$ converges to $\lambda$ vaguely in probability, i.e. for every $\varphi$ of $C_{0}(X) \quad \mathbb{E}\left[\left\langle\varphi, \xi_{t}\right\rangle \mid \xi_{0}\right]$ converges to $\langle\varphi, \lambda\rangle$ in probability.

Proof. By Proposition 1.7 and Poisson properties we have

$$
\mathbb{E}\left[\left(\mathbb{E}\left[<\varphi, \xi_{t}>\mid \xi_{0}\right]-\langle\varphi, \lambda>)^{2}\right]=\left\langle\left(T_{t} \varphi\right)^{2}, \lambda\right\rangle=\left\|T_{t} \varphi\right\|_{L^{2}(X, \lambda)}^{2} .\right.
$$

Therefore Proposition 2.6 follows from Proposition 2.5.

Remark 2.7. If $\left\{T_{t}\right\}$ has no finite invariant measure, the corresponding equilibrium process is metrically transitive.

Remark 2.8. If the equilibrium process associated with $\left[\left\{T_{t}\right\}, \lambda\right]$ is metrically transitive and $\left\{T_{t}\right\}$ are symmetric on $L^{2}(X, \lambda)$, then it is purely non-deterministic.

Remark 2.9. The equilibrium process associated with uniform motions on $R^{n}$ are mixing, but not purely non-deterministic. However the equilibrium processes associated with all the additive processes on $R^{n}$ except uniform motions are purely non-deterministic.

Remark 2.10. Let $\left(\Omega, \mathscr{F}, P_{x},\left\{x_{t}\right\}_{t \geq 0}\right)$ be a Hunt Markov process corresponding to $\left\{T_{t}\right\}$. If the equilibrium process associated with $\left[\left\{T_{t}\right\}, \lambda\right]$ is metrically transitive, for almost all $x$ (w.r.t. $\lambda$ ) and any compact subset $K, \quad P_{x}\left[\omega ; \tau_{K^{c}}<+\infty\right]=1$, where $\tau_{K^{r}}$ denote the first hitting time for $K^{c}$.

\section{§3. The Bernoulli Property of the Shiffs Fillow}

It is easy to see that $\left\{\Theta_{t}\right\}_{-\infty<t<\infty}$, which is defined by (1.4) in $\S 1$, is a flow on the probability space $\left(\Omega=\mathscr{K}(\mathscr{W}), \mathbb{B}, \mathbb{P}=\Pi_{Q}\right)$. So, we discuss the Bernoulli property in the strong sense of the flow $\left\{\Theta_{t}\right\}_{-\infty<t<\infty}$.

Definition 3.耳. $\left(\Omega, \mathbb{B}, \mathbb{P} ;\left\{\Theta_{t}\right\}_{-\infty<t<\infty}\right)$ is called Bernoulli flow if it 
satisfies the following conditions;

(3.1) There exists a system of $\sigma$-subfields $\left\{\zeta_{r}^{s}\right\}, r<s$, of $\mathbb{B}$ which satisfies

(i) $\Theta_{t} \cdot \zeta_{r}^{s}=\zeta_{r+t}^{s+t} \quad$ for every $r<s$ and $\iota$,

(ii) $\zeta_{r}^{t}=\zeta_{r}^{s} \vee \zeta_{s}^{t} \quad$ for $r<s<t$,

(iii) $\zeta_{r}^{s}$ and $\zeta_{s}^{t}$ are mutually independent for $r<s<t$,

(iv) $\underset{r<s}{\vee} \zeta_{r}^{s}=\quad\left(\bmod . \mathbb{P}^{2}\right)$

The following lemma is essentially due to $H$. Tanaka, and is a generalization of the Sinai-Volkoviskii's result on the $K$-property of the ideal gas model [4].

Lemma 3.2. Suppose that there exists a real measurable function $\tau(f)$ on the $\sigma$-finite measure space $\left(\mathscr{V}, \mathscr{B}_{\mathscr{W}}, Q\right)$ such that for almost all $f(Q)$

(a) $-\infty<\tau(f)<+\infty$

(b) $\tau(f)=t+\tau\left(\theta_{t} f\right) \quad$ for all $t$ of $\left.R^{1} \cdot{ }^{6}\right)$

Then, $\left(\Omega, \mathbb{P}, \mathbb{P} ;\left\{\Theta_{t}\right\}_{-\infty<t<\infty}\right)$ is a Bernoulli flow.

Proof. May assume every $f$ of $\mathscr{W}$ satisfies the conditions (a), (b). Put $\mathscr{W}_{r}^{s}=\{f ;-r \geqq \tau(f)>-s\}$. Then $\theta_{t} \mathscr{W}_{r}^{s}=\{f ;-r-t \geqq \tau(f)>-s-t\}$ by the condition (b). Obviously we have

$$
\theta_{t} \mathscr{W}_{r}^{s}=\mathscr{W}_{r+t}^{s+t}, \quad \bigcup_{r<s} \mathscr{W}_{r}^{s}=\mathscr{W}
$$

So, we denote by $\zeta_{r}^{s}$ the $\sigma$-subfield $\mathscr{G}\left(\mathscr{W}_{r}^{s}\right)$ which is generated by $\{\omega ; \omega(A)$ $=n\}, n \geqq 0, A \in \mathscr{B}_{\mathscr{Y}}, A \subset \mathscr{W}_{r}^{s}$. Noting $\Theta_{t}\{\omega ; \omega(A)=n\}=\left\{\omega ; \omega\left(\theta_{t} A\right)=n\right\}$, we can see $\zeta_{r+t}^{s+t}=\Theta_{t} \cdot \zeta_{s}^{r}=\mathscr{G}\left(\theta_{t} \mathscr{W}_{r}^{s}\right)$. Therefore $\zeta_{r}^{s}$ satisfies the conditions (i) (iv) in Definiton 3.1 by Lemma 1.3 .

$\mathbb{P}_{\text {roposition }}$ 3.3. Suppose that $\left\{T_{t}\right\}$ is transient in following sense; $\int_{0}^{\infty}\left(T_{t} \varphi, \varphi\right)_{L^{2}(X, \lambda)} d t<+\infty$ for every $\varphi$ of $C_{0}^{+}(X)$. Then, ( $\left.\Omega, \mathbb{B}, \mathbb{P} ;\left\{\Theta_{t}\right\}\right)$ is a Bernoulli flow.

Proof. First, we will show

6) Such a random time $\tau(w)$ is called $L$-time which was introduced by M. Nagasawa [7]. 


$$
\int_{-\infty}^{\infty} \varphi\left(f_{s}\right) d s<+\infty \text { for almost all } f(Q) \text { for every } \varphi \text { of } C_{0}^{+}(X)
$$

For any $\varphi$ and $\psi$ of $C_{0}^{+}(X)$,

$$
\begin{aligned}
\int_{\mathscr{V} L}\left[\int_{-\infty}^{\infty} \varphi\left(f_{s}\right) d s \cdot\right. & \left.\psi\left(f_{0}\right)\right] Q(d f)=\int_{\mathscr{W}}\left[\int_{0}^{\infty} \varphi\left(f_{s}\right) d s \psi\left(f_{0}\right)\right] Q(d f) \\
& +\int_{\mathscr{W}}\left[\int_{-\infty}^{0} \varphi\left(f_{s}\right) d s \psi\left(f_{0}\right)\right] Q(d f) \\
& =\int_{0}^{\infty}\left(T_{s} \varphi, \psi\right)_{L^{2}(X, \lambda)} d s+\int_{0}^{\infty}\left(\varphi, T_{s} \psi\right)_{L^{2}(X, \lambda)} d s<+\infty
\end{aligned}
$$

Therefore (3.3) holds.

Next, choose a countable sequence $\left\{\varphi_{n}\right\}$ of $C_{0}^{+}(X)$ such that $\bigcup_{n}\{x \in X$; $\left.\varphi_{n}(x)>0\right\}=X$. Putting $\mathscr{W}_{1}=\left\{f ; 0<\int_{\infty-}^{\infty} \varphi_{1}\left(f_{s}\right) d s<+\infty\right\}, \theta_{t} \mathscr{W}_{1}=\mathscr{W}_{1}$. And define $\mathscr{W}_{n+1}$ by $\left\{f ; 0<\int_{-\infty}^{\infty} \varphi_{n+1}\left(f_{s}\right) d s<+\infty\right\} \backslash \mathscr{W}_{n}$. Thus we have a sequence of disjoint subsets of $\mathscr{W}$ which are $\left\{\theta_{t}\right\}$-invariant. So define $\tau(f)=\sup \left\{t ; \int_{-\infty}^{t} \varphi_{n}\left(f_{u}\right) d u \leqq \frac{1}{2} \int_{-\infty}^{\infty} \varphi_{n}\left(f_{u}\right) d u\right\}$ if $f \in \mathscr{W}_{n}$. Then we have $\{f ;-\infty<\tau(f)<+\infty\}=\cup_{n} \mathscr{W}_{n}=\mathscr{W}(\bmod . Q)$, and if $f \in \mathscr{W}_{n} \quad \theta_{s} f \in \mathscr{W}_{n}$ and $\tau\left(\theta_{s} f\right)=\sup \left\{t ; \int_{-\infty}^{t} \varphi_{n}\left(f_{u+s}\right) d u \leqq \frac{1}{2} \int_{-\infty}^{\infty} \varphi_{n}\left(f_{u}\right) d u\right\}=\tau(f)-s$. Therefore $\tau(f)$ satisfies the conditions of Lemma 3.2.

Remark 3.4. The equilibrium process $\left\{\xi_{t}\right\}$ induces a factor flow of $\left\{\Theta_{t}\right\}$. Since a Bernoulli flow $\left\{\Theta_{t}\right\}$ in the sense of (3.1) is a Bernoulli flow in the weak sense (i.e. the automorphism $\Theta_{t}$ is Bernoulli for each $t \neq 0$ ), the shift flow induced by $\left\{\xi_{t}\right\}$ is also a Bernoulli flow in the weak sense by the theorem of Ornstein. [2]. But, perhaps, it may be a Bernoulli flow in the sense of (3.1).

Remark 3.5. In the ideal gas model of Sinai-Volkoviskii [4], the path space $\mathscr{W}$ is identified to $R^{n} \times R^{n}$ and $\zeta_{r}^{s}$ in (3.1) is the $\sigma$-algebra generated by the functions $\omega \backsim \rightarrow \omega(E), E \subset V_{r}^{s}$, where $V_{r}^{s}=\left\{f=\left(q_{1}, \ldots, q_{n}, v_{1}\right.\right.$, $\left.\left.\ldots, v_{n}\right) \in R^{n} \times R^{n} \mid-r \geqq \sum_{1}^{n} q_{i} v_{i} \geqq-s\right\}$. In this case the function $\tau(f)$ in Lemma 3.2 is given by the last exit time of the set $V_{0}^{\infty}$ for each $f \in R^{n} \times R^{n}$. 


\section{§4. A Centrall Limit Theorem}

Finally we will prove a central limit theorem related to the equilibrium process. Denote by $G \varphi(x)=\int_{0}^{\infty} T_{t} \varphi(x) d t$ if the integral is well-defined.

Proposition 4.1 . Consider any function $\varphi \in L^{2}(X, \lambda)$ which satisfies $(G|\varphi|,|\varphi|)_{\left(L^{2} X, \lambda\right)}<+\infty$, and $(G(|\varphi| G|\varphi|),|\varphi|)_{L^{2}(X, \lambda)}<+\infty$.

Then

$$
\begin{gathered}
\lim _{t \rightarrow \infty} \mathbb{P}\left[\omega ; \alpha<\frac{\int_{0}^{t}<\varphi, \xi_{s}>d s-t<\varphi, \lambda>}{\sqrt{2(\varphi, G \varphi)_{L^{2}(\lambda)^{\bullet} t}}}<\beta\right] \\
=\frac{1}{\sqrt{2 \pi}} \int_{\alpha}^{\beta} e^{-\frac{x^{2}}{2}} d x \text { for }{ }^{\forall} \alpha<{ }^{\forall} \beta .
\end{gathered}
$$

Proof. It suffices to show

$$
\begin{aligned}
\lim _{t \rightarrow \infty} \mathbb{E} & {\left[\exp i z \frac{1}{\sqrt{t}}\left(\int_{0}^{t}<\varphi, \xi_{s}>d s-t<\varphi, \lambda>\right)\right] } \\
& =\exp \left(-z^{2}(\varphi, G \varphi)_{L^{2}(X, \lambda)}\right) .
\end{aligned}
$$

$\Phi(f)=\int_{0}^{t} \varphi\left(f_{s}\right) d s$ is a function on $\mathscr{W}$ and satisfies $\langle\Phi, \omega\rangle=\int_{0}^{t}\left\langle\varphi, \xi_{s}(\omega)\right\rangle d s$. $\mathbb{E}\left[\exp i z \int_{0}^{t}\left\langle\varphi, \xi_{s}>d s\right]=\mathbb{E}\left[e^{i z<\omega, \omega\rangle}\right]=\exp \int_{\mathscr{\mathscr { C }}}\left(e^{i z \Phi(f)}-1\right) Q(d f)\right.$ by Lemma 1.2. Thus we have

$$
\begin{aligned}
& \mathbb{E}\left[\exp i z \frac{1}{\sqrt{t}}\left(\int_{0}^{t}\left\langle\varphi, \xi_{s}>d s-t<\varphi, \lambda>\right)\right]\right. \\
& =\exp \int_{\mathscr{\mathscr { V }}}\left(e^{\frac{i z}{\sqrt{t}} \Phi(f)}-1-i z \sqrt{t}<\varphi, \lambda>\right) Q(d f) .
\end{aligned}
$$

Noting $e^{i x}-1-i x-\frac{(i x)^{2}}{2}=O\left(|x|^{3}\right)$,

$$
\int_{\mathscr{V}}\left(e^{\frac{i z}{\sqrt{t}} \Phi(f)}-1-i z \sqrt{t}<\varphi, \lambda>\right) Q(d f)
$$




$$
\begin{aligned}
= & \left(\frac{i z}{\sqrt{t}}-\int_{\mathscr{Y}} \Phi(f) Q(d f)-i z \sqrt{t}<\varphi, \lambda>\right)+-\frac{1}{2} \int_{\mathscr{F}}\left(\frac{i z}{\sqrt{i}}-\Phi(f)\right)^{2} Q(d f) \\
& +O\left(\left|\int_{\mathscr{H}}\left(\frac{z}{\sqrt{t}} \Phi(f)\right)^{3} Q(d f)\right|\right) .
\end{aligned}
$$

The first term vanishes because of $\int_{\mathscr{F} \cdot} \Phi(f) Q(d f)=\int_{0}^{t}\left[\int_{\mathscr{\mathscr { V }}} \varphi\left(f_{s}\right) Q(d f)\right] d s$ $=t\langle\varphi, \lambda\rangle$.

$$
\begin{aligned}
\int_{\mathscr{F}} \Phi(f)^{2} Q(d f) & =\int_{0}^{t} d u \int_{0}^{t} d s \int_{\mathscr{F}} \varphi\left(f_{s}\right) \varphi\left(f_{u}\right) Q(d f) \\
& =2 \int_{0}^{t} d s \int_{0}^{s} d u \int_{\mathscr{Y}} \varphi\left(f_{s}\right) \varphi\left(f_{u}\right) Q(d f) \\
& =2 \int_{0}^{t} d s \int_{0}^{s} d v\left(\varphi, T_{v} \varphi\right)
\end{aligned}
$$

Therefore the second term of the right-hand side of (4.3) converges to $-z^{2}(G \varphi, \varphi)_{L^{2}(X, \lambda)}$. By the similar calculation,

$$
\begin{aligned}
\left|\int \Phi(f)^{3} Q(d f)\right| & =6\left|\int_{0}^{t} \int_{0}^{s} \int_{0}^{s-u}\left(T_{v} \varphi T_{u} \varphi, \varphi\right)_{L^{2}(\lambda)} d u d v d s\right| \\
& \leqq 6 t(G(|\varphi| G|\varphi|),|\varphi|)_{L^{2}(\lambda)} .
\end{aligned}
$$

Therefore the third term converges to zero. Thus, we can complete the proof of Proposition 4.1.

\section{Referemces}

[1] Doob, J.L., Stochastic processes, Wiley, New York, (1953).

[2] Ornstein, D.S., Factors of Bernoulli shifts are Bernoulli shifts, Adv. in Math. 5, (1970), 349-364.

[3] Port, S., Equilibrium processes, Trans. Am. Math. Soc., 124, (1966). 168-184.

[4] Sinai Y.G. and Volkoviskii, K.L., Ergodic properties of the ideal gas with infinitely many degrees of freedom, Funl. Anal. and its Appl., No. 3, (1971), 19-21 (in Russian).

[5] Spitzer, F., Random processes defined through the interaction of an infinite many particle system, Lecture Note in Mathematics, No. 89, Springer, (1969), 201-223.

[6] Totoki, H., A class of special flow, Z. Wahr. und verw. Geb., 15 (1970), 157-167.

[7] Nagasawa, M., The time reversion of Markov processes, Nagoya Math. Jour., 24 (1964), 177-204. 